\title{
An Evaluation of Image Quality Metrics for Scanning Electron Microscopy
}

\author{
Matthew D. Zotta ${ }^{1,2}$, Yukun Han $^{2}$, Matthew D. Bergkoetter ${ }^{3}$, and Eric Lifshin ${ }^{1}$ \\ ${ }^{1}$ College of Nanoscale Science and Engineering, SUNY Polytechnic Institute, Albany, NY, USA \\ ${ }^{2}$ Nanojehm Inc., Albany, NY, USA \\ ${ }^{3}$ The Institute of Optics, University of Rochester, Rochester, New York, USA
}

It has been previously demonstrated that SEM image quality and resolution can be improved by a combination of deconvolution and regularization using the point spread function (PSF) of a focused electron beam to produce a restored image [1]. One benefit of this technique is that the performance of a less expensive thermionic source SEM approaches that of a more costly Schottky or FEG instrument. If the restored image is an attempt to duplicate an image taken with a high performance microscope, then it is important to assess how closely the former matches the later.

Metrics of SEM image quality and resolution associated with direct comparison of two images, where one is a reference image, include determining the mean square error (MSE) [2], the peak signal to noise ratio (PSNR) [2] and differences between contrast transfer functions (CTF) [3]. Quantitative comparisons between images, however, are in no way limited to SEM micrographs. In this paper an image quality assessment metric known as the mean structural similarity index (MSSIM) [4] is applied to SEM micrographs. This particular method was developed to assess image degradation associated with compression methods such as JPEG. A key feature of this approach is that it recognizes the fact that two images could have the same MSE, but may have totally different responses to the human visual system (HVS). Specifically, this method involves calculating the luminance, contrast and structural factors independently, and then recombining them into a single value for simplified evaluation.

In this study, a high quality (pixel level resolution SEM image) is intentionally subjected to several perturbations such as varying the brightness and contrast and the amount of noise, vibrations and blurring. The different metrics discussed above were then calculated as a function of the degree of alteration. Figure 1a shows the reference image. Figure $1 \mathrm{~b}-\mathrm{f}$ show examples of specific mid-range levels of distortion associated with each type of alteration as well as the MSE, PSNR, and MSSIM values for each. It can be seen that for comparable values of MSE and PSNR, the MSSIM can vary significantly. Figure 2 shows the CTF curves for each of the images in Figure 1 and illustrates the change in the curves for each type of distortion.

The findings of this study are being applied both to the selection of regularization models for image restoration and also selecting optimal parameters for the functional minimization by specific models. [5] 


\section{References:}

[1] Lifshin, E. et al, Microscopy and Microanalysis 20 (01) (2014), p. 78-89.

[2] Gonzalez, R. C. in “Digital Image Processing”, (Addison-Wesley, New York), p. 354.

[3] Joy, D. C. et al, Proc. SPIE 3998, Metrology, Inspection and Process Control for Microlithography XIV (2000) p. 108.

[4] Wang, Z, IEEE Transactions on Image Processing, 13 (04) (2004), p. 600-612.

[5] The authors wish to thank Mr. Jeffrey Moskin, President of Nanojehm and NSF SBIR 1519678 for supporting this research.

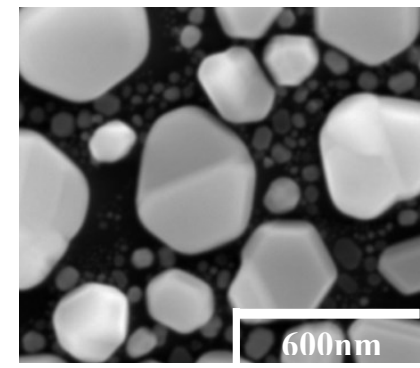

a. $\mathrm{MSE}=0, \mathrm{PSNR}=\mathrm{Inf}$, $\mathrm{MSSIM}=1$

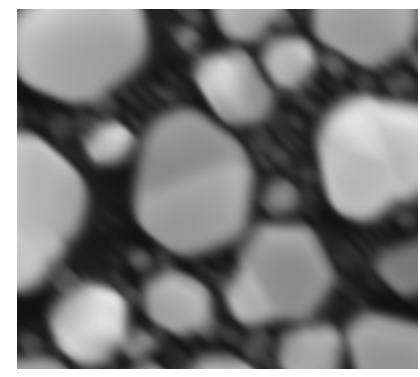

d. $\mathrm{MSE}=431, \mathrm{PSNR}=21.82$, $\mathrm{MSSIM}=0.815$

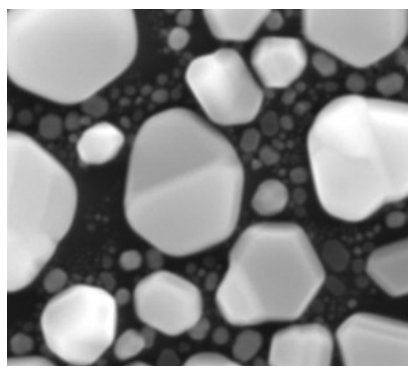

b. $\mathrm{MSE}=416, \mathrm{PSNR}=21.97$, MSSIM $=0.917$

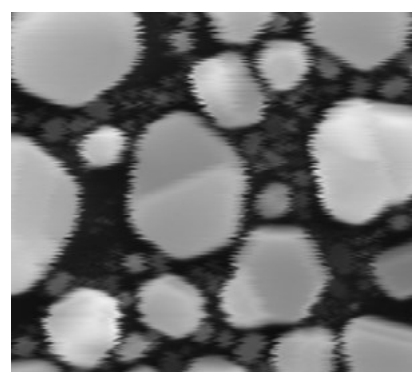

e. $\mathrm{MSE}=400, \mathrm{PSNR}=22.15$, $\mathrm{MSSIM}=0.683$

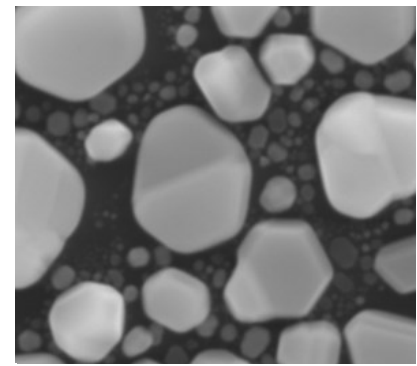

c. $\mathrm{MSE}=490, \mathrm{PSNR}=21.26$, MSSIM $=0.868$

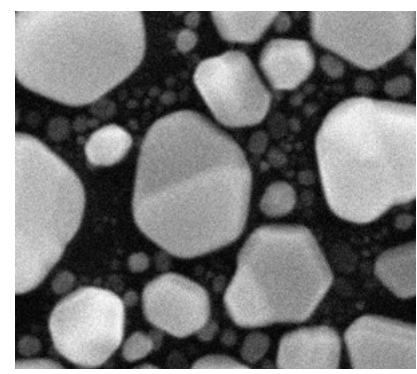

f. $\mathrm{MSE}=460, \mathrm{PSNR}=21.53$, $\mathrm{MSSIM}=0.220$

Figure 1. (a) Ideal reference image of Au-C Pella sample. (b-f) Reference image perturbed by: (b) varying brightness, (c) varying contrast range, (d) convolution with asymmetric PSF, (e) random line shifts due to vibrations with a given amplitude, (f) Gaussian noise.

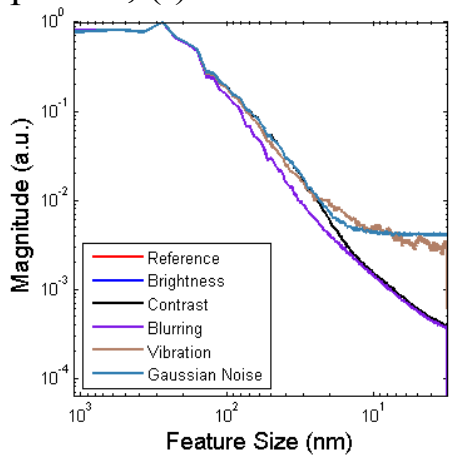

Figure 2. The CTF curves for the images shown in Figure la-f. As the CTF curve is unaffected by contrast and brightness, the "Reference", "Brightness", and "Contrast" curves trace back directly on top of one another. However, this is not the case when the reference image is distorted by blurring (Figure 1d), vibrations (Figure 1e) and Gaussian noise (Figure 1f). In these cases, the curves are altered by changing the slope of the curves in the midrange of feature sizes or by changing the perceived noise floor or by a combination thereof. 